\title{
Simulation of coupled nonlinear electromagnetic heating with the Green element method
}

\author{
A. E. Taigbenu \\ School of Civil and Environmental Engineering, \\ University of the Witwatersrand, Johannesburg, South Africa
}

\begin{abstract}
The nonlinear coupled differential equations that govern the problem when materials are electrically heated are solved with a flux-based Green element formulation that has significantly enhanced computational features in comparison to previous Green element formulations. The flux-based Green element formulation takes advantage of the ability of the boundary element theory to correctly calculate the normal derivative of the primary variable by implementing the theory in a finite element sense so that enhanced accuracy is achieved with coarse discretization. The complete solution information (temperature, electric potential and their normal derivatives) are made available by the flux-based formulation in each element so that refined solutions at any point, when needed, are calculated using only the element in which the point is located. The closure problem associated with having more unknowns than discretized equations at internal nodes is addressed in a novel manner by a compatibility relation for the normal derivatives of the primary variable that has universal appeal. The computational accuracy of the flux-based Green element formulation is demonstrated with a numerical example of nonlinear electromagnetic heating problem.
\end{abstract}

Keywords: electromagnetic heating, nonlinear diffusion-advection, nonlinear Poisson equation, Green element method.

\section{Introduction}

The food and related industries are very interested in addressing the problems associated with the heating of food substances by electrical currents. Of 
particular interest is the distribution of the temperature field (indicative of the effectiveness of the applied heat) that is generated for a given electric potential that is applied to the food material. For the temperature field, the governing equation closely resembles the diffusion-advection-reaction equation that has many applications in many engineering production processes. Its nonlinear nature poses a challenge especially when the advective term is dominant and the equation then has a predominantly hyperbolic nature. This equation is couple with the nonlinear electromagnetic equation which essentially has an elliptic character.

These equations were traditionally solved by the finite difference and finite element methods. A boundary element solution has been provided by Crann et al. [1] who used the Laplace transform formulation to simplify the temporal derivative and the dual reciprocity method to ensure that the solution is carried out only on the boundary. That approach, referred to as the Laplace transform dual reciprocity method (LTDRM), is closely similar to that of Satravaha and Zhu [2] for the solution of nonlinear heat conduction problems. Linearization of the differential equations has to be done to be able to apply the Laplace transform dual reciprocity BEM. Here the Green element method (GEM), which retains the normal derivatives at every nodal point, is used to solve the electromagnetic heating problem [3-5]. It is therefore referred to as the flux-based GEM. The integral equations that result from the application Green's identity are solved in each element. The integrations are evaluated analytically, and the only approximation that is required is done when interpolating the primary variable and its normal derivative in the element. High level of accuracy is thus achieved with coarse discretization of the computational domain and this compensates for the large number of degrees of freedom at each node. The closure problem at the internal nodes that is as a result of a fewer number of integral equations than unknowns is resolved in a novel manner by generating an additional equation from numerically implementing the integration of the normal fluxes around the internal node. An example solved by the LTDRM of Crann et al. [1] which employed 40 nodes is solved by the flux-based GEM with 10 nodes to achieve comparable accuracy.

\section{Governing equations}

The electromagnetic heating problem that is addressed in this paper is governed by the coupled nonlinear equations that are given by Please et al. [6]

$$
\nabla \cdot(k \nabla T)=\frac{\partial}{\partial t}(\alpha T)+\mathbf{v} \cdot \nabla(\alpha T)-\sigma|\nabla \phi|^{2}
$$

and

$$
\nabla \cdot(\sigma \nabla \phi)=0
$$

where $T$ and $\phi$ represent the temperature and electric potential fields of the medium on which ohmic heating is applied. The material properties of the medium are: $k$ is the thermal conductivity and $\sigma$ is the electrical conductivity which are both dependent on the temperature field, $\alpha$ is the heat capacity, 
$\mathbf{v}=\mathbf{i} u+\mathbf{j} v$ is the velocity field in two dimensions. The solution to eqns (1) and (2) in a coupled manner can be achieved when the boundary and initial conditions are specified. The first-, second- and third-type boundary conditions are admissible, while the condition for the temperature and potential fields are assumed known at the initial time $t_{0}$. Eqns (1) and (2) are rewritten as Poisson-type equations:

$$
\begin{gathered}
\nabla^{2} T=-\nabla K \cdot \nabla T+\frac{1}{k}\left[\frac{\partial}{\partial t}(\alpha T)+\mathbf{v} \cdot \nabla(\alpha T)-\sigma|\nabla \phi|^{2}\right] \\
\nabla^{2} \phi=-\nabla \Phi \cdot \nabla T
\end{gathered}
$$

where $K=\ln k$ and $\Phi=\ln \sigma$. The solutions to eqns (3) and (4) are achieved in a Green element sense.

\section{Green element formulation}

The Green element formulation that is employed for the solution of the coupled equations (3) and (4) uses the fundamental solution $G=\ln \left(r-r_{i}\right)$ to the Laplacian operator $\nabla^{2} G=\partial\left(r-r_{i}\right)$ in the infinite space to derive integral equations within a spatial element $\Omega^{e}$ with closed boundary $\Gamma^{e}$ that constitutes one of the elements used in discretizing the entire computational region $\Omega$. The integral equations are given by [7]

$$
\begin{aligned}
& -\lambda T_{i}+\int_{\Gamma^{e}}(T \nabla G \cdot \mathbf{n}-G \nabla T \cdot \mathbf{n}) d s \\
& +\iint_{\Omega^{e}} G\left[-\nabla K \cdot \nabla T+\frac{1}{k}\left(\frac{\partial(\alpha T)}{\partial t}+\mathbf{v} \cdot(\alpha T)-\sigma|\nabla \phi|^{2}\right)\right] d A=0 \\
& \quad-\lambda \phi_{i}+\int_{\Gamma^{e}}(\phi \nabla G \cdot \mathbf{n}-G \nabla \phi \cdot \mathbf{n}) d s-\iint_{\Omega^{e}} G(\nabla \Phi \cdot \nabla \phi) d A
\end{aligned}
$$

where $\lambda$ is the nodal angle from integrating the dependent variable with the Dirac delta function in a Cauchy sense, and $\mathbf{n}$ is the outward pointing normal on the elemental boundary. It should be noted that so far no approximation whatsoever has been introduced in the formulation in arriving at eqns (5) and (6). Approximations are introduced by prescribing an interpolation for the distribution of the dependent variable and their normal derivatives in the element. The Lagrange-type interpolation functions are used. Using either linear triangular or linear rectangular elements and carrying out the integrations results in the matrix equations 


$$
\begin{array}{r}
R_{i j} T_{j}+L_{i j}\left(\frac{q}{k}\right)_{j}+\mathrm{X}_{i m j}\left[\alpha_{m}\left(\frac{u}{k} T\right)_{j}+K_{m}\left(\frac{q_{x}}{k}\right)_{j}\right]+\mathrm{Y}_{i m j}\left[\alpha_{m}\left(\frac{v}{k} T\right)_{j}+K_{m}\left(\frac{q_{y}}{k}\right)_{j}\right] \\
+S_{i m j}\left[\left(\frac{1}{k}\right)_{m} \frac{d(\alpha T)_{j}}{d t}-\left(\frac{\alpha u}{k}\right)_{m}\left(\frac{q_{x}}{k}\right)_{j}-\left(\frac{\alpha v}{k}\right)_{m}\left(\frac{q_{y}}{k}\right)_{j}-\left(\frac{\sigma}{k}\right)_{m}|\nabla \phi|_{j}^{2}\right]=0 \\
R_{i j} \phi_{j}+L_{i j}\left(\frac{p}{k}\right)_{j}+\mathrm{X}_{i m j} \Phi_{m}\left(\frac{p_{x}}{k}\right)_{j}+\mathrm{Y}_{i m j} \Phi_{m}\left(\frac{p_{y}}{k}\right)_{j}=0
\end{array}
$$

where

$$
\begin{aligned}
& R_{i j}=\int_{\Gamma} N_{j} \nabla G \cdot \mathbf{n} d s-\delta_{i j} \lambda, \quad L_{i j}=\int_{\Gamma} N_{j} G d s, \\
& \mathrm{X}_{i m j}=\iint_{\Omega} G\left(\frac{\partial N_{m}}{\partial x} N_{j}\right) d A, \quad \mathrm{Y}_{i m j}=\iint_{\Omega} G\left(\frac{\partial N_{m}}{\partial y} N_{j}\right) d A, \\
& S_{i m j}=\iint_{\Omega} G\left(N_{m} N_{j}\right) d A . \\
& q=-k \nabla T \cdot \mathbf{n}, \quad q_{x}=-k \nabla T \cdot \mathbf{i}, \quad q_{y}=-k \nabla T \cdot \mathbf{j} \\
& p=-\sigma \nabla \phi \cdot \mathbf{n}, \quad p_{x}=-\sigma \nabla \phi \cdot \mathbf{i}, \quad p_{y}=-\sigma \nabla \phi \cdot \mathbf{j}
\end{aligned}
$$

In eqn (9), $N_{j}$ represents the interpolation function with respect to node $j$. It should be noted that the integrations in eqn (9) are done in each element, and it is for simplicity that the index $e$ has been excluded. All the integrations are done analytically for the two types of elements, namely rectangular and triangular elements. Carrying out the matrix manipulations in eqns (7) and (8) results in two unknowns at every nodal point for each of the equations. These are the temperature $T$ and its normal flux $q$ for the first differential equation, and the electric potential $\phi$ and its normal flux $p$. Eqns (7) and (8) are aggregated for all elements used in discretizing the computational domain and simplified to

$$
\begin{gathered}
\mathbf{A}^{n} \mathbf{T}^{n+1}+\mathbf{B}^{n} \mathbf{q}^{n+1}+\mathbf{S}^{n} \frac{d \mathbf{T}^{n+1}}{d t}=0 \\
\mathbf{C}^{n} \boldsymbol{\varphi}^{n+1}+\mathbf{E}^{n} \mathbf{p}^{n+1}=0
\end{gathered}
$$

We have introduced a new index $n$, the iteration number, into eqns (11) and (12) to indicate that the elements of the matrices are evaluated with known iterates, while the unknown quantities are to be computed at the current iteration level of $n+1$. Essentially, this iteration process is the Picard algorithm. The time derivative in eqn (11) is simplified by the generalized finite difference scheme with weighting factor $\theta$ to become

$$
\theta \mathbf{A}^{n} \mathbf{T}^{n+1,2}+\omega(\mathbf{A} \mathbf{T})^{1}+\theta \mathbf{B}^{n} \mathbf{q}^{n+1,2}+\omega(\mathbf{B q})^{1}+\mathbf{S}^{n} \frac{\mathbf{T}^{n+1,2}-\mathbf{T}^{n+1,1}}{\Delta t}=0
$$

where the indices 2 and 1 , respectively, are indicative of the current time $t_{2}=t_{1}+\Delta t$ and previous time $t_{1}$, and $\omega=1-\theta$. Introducing the initial 
conditions into eqn (13), and the known boundary conditions on external boundaries into eqns (12) and (13) simplify the coupled equations to

$$
\begin{aligned}
& \mathbf{F}^{n} \mathbf{T}^{n+1,2}+\mathbf{H}^{n} \mathbf{q}^{n+1,2}=\mathbf{R} \\
& \mathbf{C}^{n} \boldsymbol{\varphi}^{n+1}+\mathbf{E}^{n} \mathbf{p}^{n+1}=\mathbf{M}
\end{aligned}
$$

where

$$
\mathbf{F}^{n}=\theta \mathbf{A}^{n}+\frac{\mathbf{S}^{n}}{\Delta t}, \quad \mathbf{H}^{n}=\theta \mathbf{B}^{n}
$$

The vectors on the right hand side of eqns (14) and (15) are known; their values come from the prescribed boundary and initial conditions. The solution procedure within each time step is herein outlined.

(i) For the known constitutive relations for $k$ and $\sigma$, and a known distribution of the temperature field $\left\{\mathbf{T}^{n, 2}, \mathbf{q}^{n, 2}\right\}$, the matrix equation (14) is solved to obtain updated values $\left\{\mathbf{T}^{n+1,2}, \mathbf{q}^{n+1,2}\right\}$.

(ii) Those updated solutions from (i) are used in eqn (15) to solve for the electric potential field $\left\{\boldsymbol{\varphi}^{n+1}, \mathbf{p}^{n+1}\right\}$.

(iii) The mean deviation of the iterates from the temperature and potential fields is calculated and compared with a prescribed accuracy tolerance $\varepsilon$. Convergence is said to be achieved when the mean deviation is less than $\varepsilon$. If convergence is not attained, steps (i) through (iii) are implemented using the refined solution iterates.

(iv) When convergence within a time step has been met, another time increment is made and the above three steps are then repeated.

\section{Compatibility of the internal fluxes}

Because the current flux-based Green element formulation calculates the dependent variable and as well as its normal derivative (referred to as the flux) at each nodal point, the number of generated integral equations is short by one the number of degrees of freedom at internal nodes. This is not the case with the external nodes where the prescribed boundary conditions make up for the shortfall in the number of generated discrete equations. This is the closure problem that has been recognized in boundary element circles. In the past one approach of resolving the closure problem has been to artificially create additional nodes and relocate them by small distances from the original location of the internal node along the internal segments [8]. Such an approach is a numerical artefact that usually reconfigures the geometry at the internal nodes to suit the numerical formulation. That approach is not followed here but rather an additional equation is generated to make up for the shortfall. Two previous presentations on this additional equation indicated that this additional equation is statement of the continuity of the normal fluxes at the internal node $[4,5]$. We have now found this to be in error, and special acknowledgement goes to Dr. Elliot who prompted us to re-examine that statement. We have now carried 
out a rigorous proof of this compatibility condition of the internal normal fluxes. It is too lengthy to present here. The proof will be presented in details in manuscripts that are currently being prepared. Suffice to say that the proof is based on integrating the normal derivative of the primary variable around a circle of small radius centred at the internal node. In the limit as the radius tends to zero, the integral equals the value of discontinuity of the primary variable at the internal point. Where no discontinuity exists, the integral equals zero. This can be stated as

$$
\oint k \frac{\partial T}{\partial n} d n=T^{+}-T^{-}=0
$$

To implement eqn (17) numerically,

$$
\oint k \frac{\partial T}{\partial n} d n=-\underset{\zeta \rightarrow 0}{\operatorname{Limit}} 2 \pi \int_{0}^{2 \pi} q d \beta \approx \sum_{i=1}^{M} q_{i} \Delta \beta_{i}=0
$$

where $M$ is the number of elements at the internal node, $\zeta$ is the radius of the circle at the internal node (See Figure 1). One may ask why excellent results were obtained in the previous publications with a wrong compatibility condition. The reason is that all the simulations were carried out with rectangular elements and, as can be observed from eqn (18), $\Delta \beta_{i}=\pi / 2, i=1,2,3,4$. We have since tested the condition of eqn (18) with triangular elements where different angles of $\Delta \beta_{i}$ are encountered and excellent results were obtained.

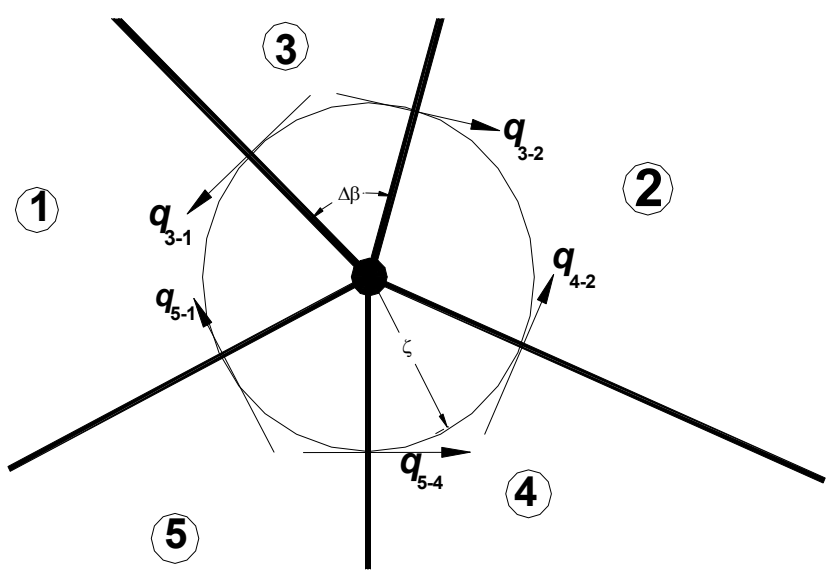

Figure 1: Normal fluxes at an internal node.

Two unique characteristics of the flux-based formulation are that it provides the complete solution information for each element, and high accuracy is achieved with coarse discretization. The latter compensates for the escalation in the number of degrees of freedom due to the evaluation of the primary variable 
and the flux at every node. The former allows for calculations of the solution at a point to involve only integrations within the element in which the point is located. No reference is made to other elements in the computational region. That represents enormous computational savings when solutions are required at points of interest other than grid points.

\section{Numerical example}

The numerical example used by Crann et al. [1] in their Laplace transform dual reciprocity boundary element formulation is also used here to validate the current flux-based Green element formulation. The example is essentially one dimensional in the spatial dimension for which the analytical solutions:

$$
T=\left(x-x^{2} / 2\right)\left(2-e^{-t}\right) \text { and } \phi=x+\left(x-x^{2}\right) e^{-t}, 0 \leq x \leq 1
$$

are proposed for the differential equations

$$
\nabla \cdot(k \nabla T)=\frac{\partial}{\partial t}(\alpha T)+\mathbf{v} \cdot \nabla(\alpha T)-\sigma|\nabla \phi|^{2}+f_{1}(x, t)
$$

and

$$
\nabla \cdot(\sigma \nabla \phi)=f_{2}(x, t)
$$

The choice of the functions $f_{1}(x, t)$ and $f_{2}(x, t)$ has been made so that the proposed solutions satisfy the differential equations. The boundary conditions are:

$$
T(x=0, t)=0, \frac{\partial T(x=1, t)}{\partial x}=0, \phi(x=0, t)=0 \text { and } \phi(x=1, t)=1
$$

The parameters for the medium and the advection velocity field are: $k=1+T$, $\sigma=1+T, \alpha=1$ and $\mathbf{v}=\mathbf{i}$. Using only four linear rectangular elements, the Green element simulations are carried out in a two dimensional domain of $1 \times 1$ so that the size of each element is $0.25 \times 1$. Effectively, 10 nodes are used to discretize the computation domain, representing a coarse discretization in comparison to the 40 nodes used by Crann et al. [1]. The fully implicit scheme with $\theta=1$ is used for the differencing in time, while the time step is varied starting with 0.025 for $0 \leq t \leq 0.1$, then 0.1 for $0.1 \leq t \leq 1$ and 0.25 for $1 \leq t \leq 5$. The accuracy tolerance value used is $\varepsilon=10^{-5}$ and convergence was achieved within 3 iterations in each time step. The spatial distributions of the temperature and electric potential fields for times of $0.1,0.5,1$, and 5 are presented in Figures 2 and 3, while their temporal distributions at $x=0.25, x=0.5$, and $x=0.75$ are presented in Figures 4 and 5 . There is good agreement between the Green element solutions and the analytical solution. It should be pointed out that the solutions at points other than grid points were generated using only the solutions obtained for the element in which the grid points are located. For instance, the solution at $x=0.1$ was carried out on the first element $\{(x, y): x \in[0,0.25], y \in[0,1]\}$. The high level of accuracy that is achieved with such a coarse grid is as a result of the fact that the only approximation in this formulation arises from the interpolation of the primary variable and its normal derivative. 


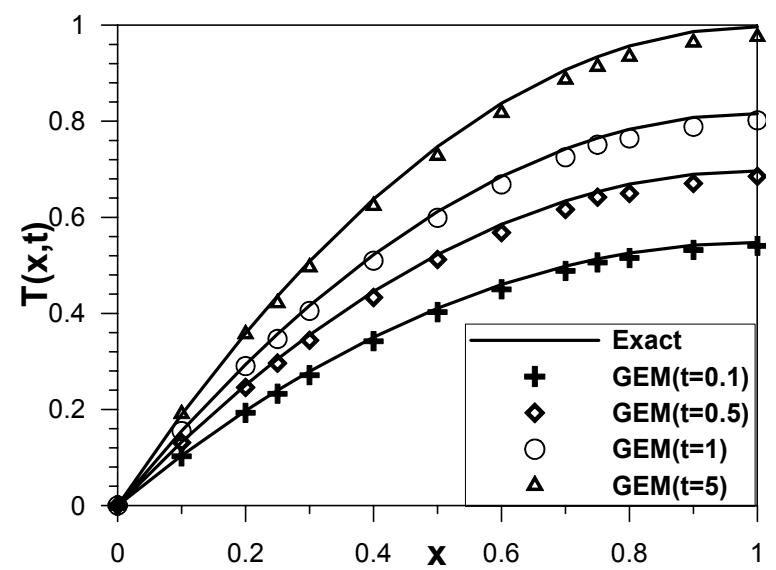

Figure 2: Analytical and GEM solutions for the spatial temperature distribution at various times.

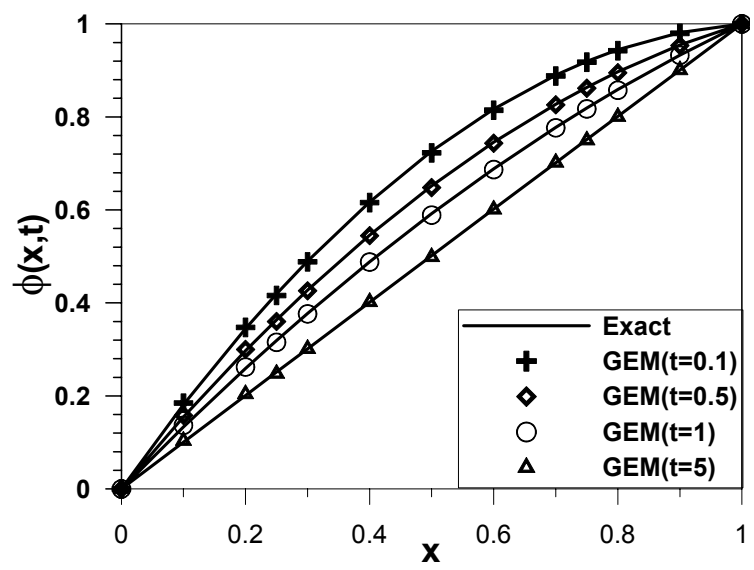

Figure 3: Analytical and GEM solutions for the spatial electric potential distribution at various times.

\section{Conclusion}

The nonlinear coupled differential equations which govern heating of food materials by electrical current have been solved using a flux-based Green element formulation that solves not only for the temperature and electric potential fields but also their normal derivatives. The high level of accuracy achieved by the formulation arises not only because the solution procedure retains the nonlinear nature of the differential equations, but also due to the fact 
that the normal derivatives of the temperature and electric potential are directly calculated. The increased number of degrees of freedom at each node is compensated by the coarse grid with which high accuracy is achieved.

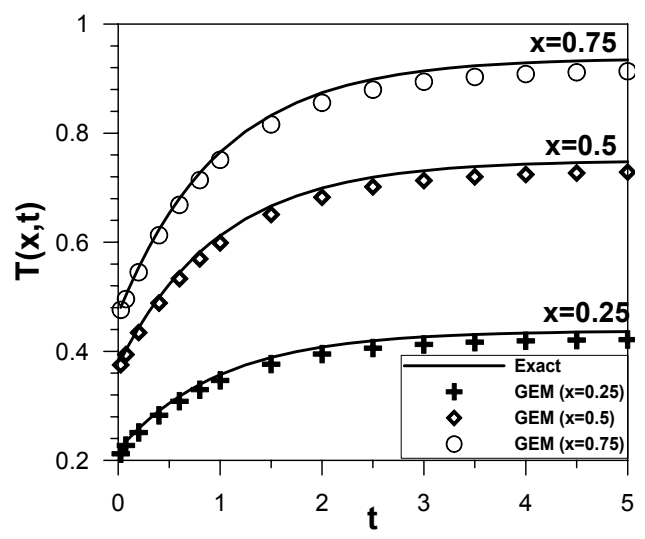

Figure 4: Analytical and GEM solutions for the temporal temperature distribution at various positions.

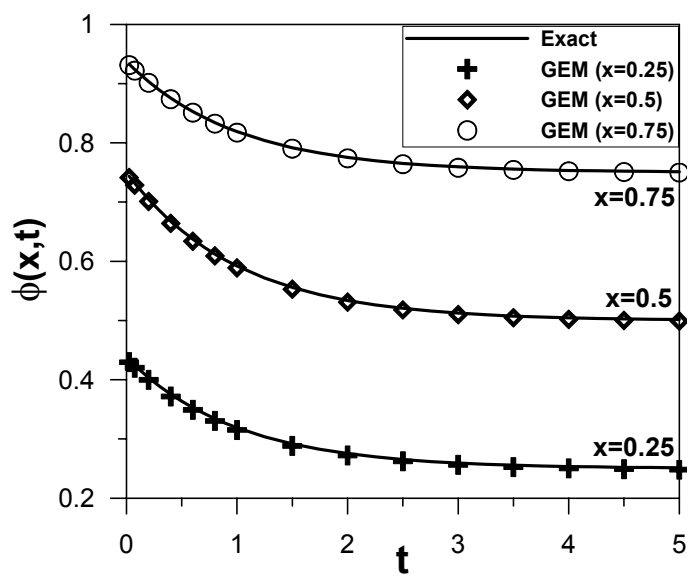

Figure 5: Analytical and GEM solutions for the temporal electric potential distribution at various positions.

The novel feature of the current Green element formulation is that the elemental solution is complete in the sense that the solution at any point in an element is obtained by carry out the boundary and domain integrations within that element. No reference is made to other elements that have been used to discretize the region. Those solutions at points other than the grid points are equally second-order accurate as those at the nodal points. In this paper, the 
current flux-based GEM has used 25\% of the grid points of LTDRM formulation of Crann et al. [1]. That is typical of the flux-based GEM which generally provides accurate solution with very coarse discretization.

\section{Acknowledgement}

Special thanks to Dr. L. Elliot of Leeds University who prompted a rethink of compatibility equation of the fluxes at the internal node at the end of the $5^{\text {th }}$ UK conference on Boundary Integral Methods in Liverpool in September 2005.

\section{References}

[1] Crann, D., Davies, A.J. \& Christianson, B., The Laplace transform dual reciprocity boundary element method for electromagnetic heating problems. Proc. In Advances in Boundary Element techniques VI, Eds. A.P. Selvadura, C.L. Tan \& M.H. Aliabadi, EC LTd, UK, pp. 229-234, 2005.

[2] Satravaha, P. \& Zhu S., An application of the LTDRM to transient diffusion problems with nonlinear material properties and nonlinear boundary conditions, App. Maths Comp., 87, pp. 127-160, 1997.

[3] Pecher, R., Harris, S.D., Knipe, R.J., Elliot L. \& Ingham D.B., New formulation of the Green element method to maintain its second-order accuracy in 2D/3D, Engrg. Anal. with Boundary Elements, 25, pp. 211-219, 2001 .

[4] Taigbenu, A.E., The flux-correct Green element method for linear and nonlinear potential flows, Proc. In Advances in Boundary Element techniques VI, Eds. A.P. Selvadura, C.L. Tan \& M.H. Aliabadi, EC LTd, UK, pp. 245-250, 2005.

[5] Taigbenu, A.E., Improvements in heat conduction calculations with fluxbased Green element method, Proc. Of the $5^{\text {th }}$ UK conf. on boundary integral methods, Ed. Ke Chen, The University of Liverpool Press, pp. 190-199, 2005.

[6] Please, C.P., Schwendenman, D.W. \& Hagan, P.S., Ohmic heating of foods during aseptic processing, IMA J. of Management Maths., 5(1), pp. 283-301, 1993.

[7] Taigbenu, A.E. The Green Element Method, Kluwer, Boston, USA, 1999.

[8] Liggett, J.A. \& Liu, P.L-F, The boundary Integral Equation Method for Porous Media Flow, George Allen \& Unwin, 1983. 